\title{
The new A-dec 500: a legend, redefined
}

A-dec are pleased to announce the launch of a completely redefined new A-dec 500, combining the reliability and longevity that dental teams expect, with access, integration, comfort and technology, to make it truly a pleasure to work with.

'As much as we want to talk about our system and how beautiful it is, and how many things it does, the dentist basically wants it to become invisible. They want to forget about it and focus on the patient,' says Tom McCleskey, Director of Core Equipment, Clinical and Product Management at A-dec.

The chair and delivery system have been re-designed to function holistically and intuitively, while remaining simple and easy to use. That attention to modern simplicity is the beauty behind the new system. With the ability to effortlessly make adjustments in mind, A-dec built in extra safeguards and responsiveness including LED coolant indicators that light up when a handpiece is lifted out of the holder. The glass touchpad uses visual, user-friendly icons to eliminate clutter and positive positioning allows for smooth, onehanded movement of the delivery system without any drift.

'It's important that dentists can rely on our equipment and not have it disappoint,' adds McCleskey. 'That's where you see peace of mind relate to reliability and quality. It's an outcome of a superior experience.'

Reliability and peace of mind are woven through every element of the new A-dec 500. Both chair and delivery system are designed to provide optimal access, allowing you to move in close and position everything within reach. Clinical instruments can be custom configured, and the control pad is fully customisable for easy navigation with the ability to add additional instruments now or later. The classic headrest and iconic ultra-thin backrest deliver consistent support for the patient from any angle, while simultaneously allowing the dentist to work ergonomically.

Highlights of the new A-dec 500 include:

- Primea Advanced Air from W\&H is the world's first air-driven, high speed handpiece with an adjustable bur speed and constant removal

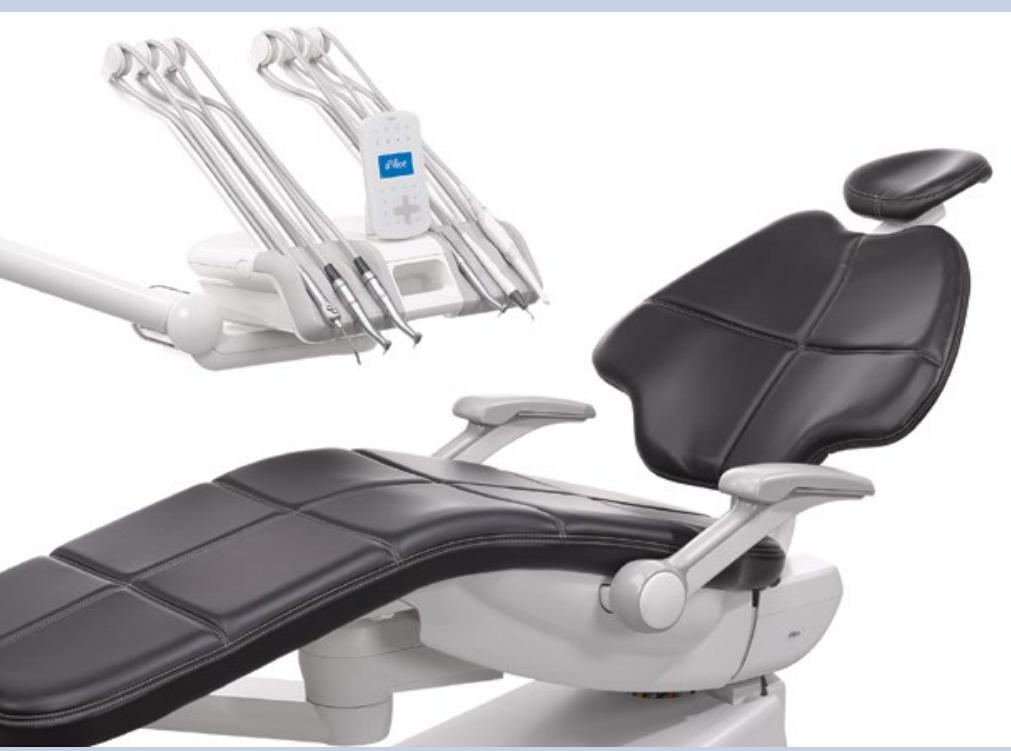

rate - even with increasing pressure. Integrated with the A-dec 500, this award-winning new technology is compact and lightweight, yet powerful and precise. A sensor in the handpiece constantly measures the rotation speed of the bur on the tooth, immediately adjusting the air supply to maintain maximum treatment efficacy at all times. With one handpiece, a complete high-speed range of procedures can be efficiently achieved, opening new possibilities for any practice

- Positionable Deluxe Plus touchpad minimises the extraneous and dynamically displays only what is necessary for the current procedure. The tilting screen and rotating arm adjust for precise viewing from multiple angles, and delivery system arm moves for right- or left-handed operators, allowing optimal visibility from either side of the control head

- LED coolant indicator lights up when a handpiece is lifted out of the holder. These individually adjustable water coolant lights identify which handpiece is in the active position, for easy adjustments without second guessing

- Automatic braking allows for effortless positioning of the delivery system with one hand - grasp the handle and the flexarm brake automatically releases. Let go, and the delivery system will stay firmly in place

- Effortlessly ambidextrous - go from left-to right-handed delivery in seconds. The centrally located handle and touchpad are reachable from either side, ensuring optimal visibility from either orientation. Dual operator settings allow access to personalised settings on shared equipment with a single touch

- Increased weight capacity up to 500 pounds $(227 \mathrm{~kg})$ to accommodate a wide range of patient size. Sturdy multi-position armrests solidly support patients during entry and exit. A rigid frame absorbs movement and optimises stability, while a powerful hydraulic cylinder guarantees smooth transitions with a 10 -year warranty

- Quicker installations and servicing - the new A-dec 500 is even more robust that its predecessor and servicing simplified to save dentists from costly downtime.

A-dec products are engineered to last, but technology is ever-changing. With a flexible, forward thinking design, the A-dec 500 perfects your practice today - and grows with it tomorrow.

For more information or to book a showroom appointment, contact A-dec on 0800233285 or email info@a-dec.co.uk.

\section{The A-dec difference}

Every product is designed with attention to detail and focus on continual improvement. A highly trained customer service team is onsite to keep practices running smoothly-even if the question is about a product made 50 years ago.

A-dec continues working for the worldwide betterment of dentistry and making a difference in the lives and practices of dental teams around the world. 